\title{
Detection of primary RGB colors projected on a screen using fNIRS
}

\author{
Xiaolong Liu \\ School of Mechanical Engineering \\ Pusan National University, 2 Busandaehak-ro, Geumjeong-gu \\ Busan 46241, Republic of Korea \\ Keum-Shik Hong* \\ School of Mechanical Engineering \\ and Department of Cogno-Mechatronics Engineering \\ Pusan National University; 2 Busandaehak-ro \\ Geumjeong-gu, Busan 46241, Republic of Korea \\ kshong@pusan.ac.kr
}

Received 27 September 2016

Accepted 8 January 2017

Published 15 February 2017

\begin{abstract}
In this study, functional near-infrared spectroscopy (fNIRS) is utilized to measure the hemodynamic responses (HRs) in the visual cortex of 14 subjects (aged 22-34 years) viewing the primary red, green, and blue (RGB) colors displayed on a white screen by a beam projector. The spatiotemporal characteristics of their oxygenated and deoxygenated hemoglobins ( $\mathrm{HbO}$ and $\mathrm{HbR}$ ) in the visual cortex are measured using a 15-source and 15-detector optode configuration. To see whether the activation maps upon RGB-color stimuli can be distinguished or not, the $t$-values of individual channels are averaged over 14 subjects. To find the best combination of two features for classification, the HRs of activated channels are averaged over nine trials. The HbO mean, peak, slope, skewness and kurtosis values during $2-7 \mathrm{~s}$ window for a given $10 \mathrm{~s}$ stimulation period are analyzed. Finally, the linear discriminant analysis (LDA) for classifying three classes is applied. Individually, the best classification accuracy obtained with slope-skewness features was $74.07 \%$ (Subject 1), whereas the best overall over 14 subjects was $55.29 \%$ with peak-skewness combination. Noting that the chance level of 3-class classification is $33.33 \%$, it can be said that RGB colors can be distinguished. The overall results reveal that fNIRS can be used for monitoring purposes of the HR patterns in the human visual cortex.
\end{abstract}

Keywords: Color detection; functional near-infrared spectroscopy; visual cortex; $t$-map; LDA classification.

${ }^{*}$ Corresponding author.

This is an Open Access article published by World Scientific Publishing Company. It is distributed under the terms of the Creative Commons Attribution 4.0 (CC-BY) License. Further distribution of this work is permitted, provided the original work is properly cited. 


\section{Introduction}

Color is a fundamental aspect of human perception and also affects the human spirit. It should come as no surprise, then, that color stimulation and detection in the visual cortex have been the subjects of long-standing study and debate. ${ }^{1-13}$ The objective of the present study is to characterize appropriate features of the hemodynamic response (HR) signals obtained from the visual cortex upon color stimuli. Five different features (i.e., mean, slope, peak, skewness, and kurtosis) of the obtained HR signals are examined in order to classify the three different color stimuli (i.e., red, green, and blue (RGB)).

Scientists have investigated visual responses via color stimuli using various modalities such as functional magnetic resonance imaging (fMRI), electroencephalography (EEG), and functional nearinfrared spectroscopy (fNIRS), among others. The works on fMRI- and EEG-based color detection are extensive and various, though the literature on fNIRS is sparse.

The work of Xiao et al. ${ }^{1}$ on cortical activation in response to chromatic stimuli revealed a topographic representation of chromaticity. They found that upon color stimulation, a cortical response is evoked in the visual cortex, and that this usually occurs as a result of the flickering of various colors with gray, specifically red-gray, green-gray, bluegray and yellow-gray combinations. Their results showed that based on color blobs in the monkey visual cortex, the red-green and red-gray flickers produced the strongest responses. Lu and $\mathrm{Roe}^{2}$ used optical imaging to map color-selective responses in $\mathrm{V} 1-\mathrm{V} 2$ and Tanigawa et al. ${ }^{3}$ in V4. Fine alignment of optical maps and cytochrome-oxidase (CO)stained tissue revealed that color domains in $\mathrm{V} 1$ strongly associate with CO blobs. They also found that color domains in V1 align along the centers of ocular dominance columns. Another fMRI study done by Goddard et al. ${ }^{4}$ showed greater blood-oxygenation-level-dependent (BOLD) activation for certain cone-opponent channels, whose finding suggests integration of those channels as early as V1. However, Brouwer and Heeger ${ }^{5}$ found that the transition from cone-opponent color coding to perceptual color coding began at V1 and that the activations were strongest at V4 and VO1. Similarly, Parkes et al., ${ }^{6}$ having measured the fMRI BOLD responses to flickering radial patterns composed of perceptual colors (red, green, yellow, blue), found that the BOLD responses evoked were similar, even though the individual differences with respect to each color pattern were sufficiently reliable to predict the color viewed. Mullen et al.,${ }^{7}$ having measured the fMRI BOLD response in the visual cortex to radial chromatic red/green and yellow/ blue grating patterns differing either in cone activation or in multiples of detection threshold, determined that the amplitude of the BOLD response was not linearly related to either measure. In their later investigation of the response of early and extra-striate visual areas to color, specifically highcontrast red-green (RG), they found that the most evident in the early visual areas (V1 and V2), but selective responses, revealed as greater adaptation between the same stimuli than cross-adaptation between different stimuli, emerged in the ventral cortex, in V4 and VO in particular. ${ }^{8}$ Laeng et al., ${ }^{9}$ working with a cohort of synaesthesia patients viewing both chromatic letters and illusory colors, measured the color differences in RGB and CIExyY color spaces, and identified a correlation between the BOLD activation and the color difference calculated using either color space. About human visual cortex research, Kuriki et al. ${ }^{10}$ applied a deriving histogram of hue-selective voxels measured using fMRI with a novel stimulation paradigm. They demonstrated that there exists a wide spectrum of hue selectivity in early visual cortex.

As for EEG research, Tang and Zhang, ${ }^{11}$ after conducting a brain-activity-based color-determination study, reported that the dispositive brain activity is a function of color energy and frequency. Rasheed and Marini, ${ }^{12}$ applying an EEG modality to the classification of RGB-color stimuli, classified three visual conditions to $84 \%, 89 \%$ and $98 \%$ accuracies with linear, polynomial, and radial basis function kernels, respectively. Alharbi et al. ${ }^{13}$ similarly evaluated a single-trial classification model for RGB-color-stimulus-evoked EEG signals, and determined that the empirical mode decomposition residual provides the most accurate, fastest, and most reliable classification (average accuracy: $88.5 \%$, execution time: $14 \mathrm{~s}$ ).

fNIRS is a noninvasive, optical imaging technique for continuous monitoring of oxygenated hemoglobin (HbO) and deoxygenated hemoglobin (HbR). ${ }^{14-33}$ It can provide both topographic ${ }^{15,16,34-36}$ and tomographic ${ }^{14,37}$ brain images. Specifically, fNIRS monitors regional cerebral blood flow (rCBF) variations by measuring, at wavelengths between 
$700 \mathrm{~nm}$ and $1000 \mathrm{~nm}$, the near-infrared-lightabsorption changes caused by concentration variations of $\mathrm{HbO}$ and $\mathrm{HbR}$, the two primary absorbing chromophores in brain capillary blood. ${ }^{24,38}$ Likewise, fNIRS has been used to measure fingeropposition activation of the cerebral cortex. ${ }^{39}$ The same finger-opposition task also has been shown to activate the primary motor cortex. A relevant previous work indicated that normal subjects, during the performance of a verbal fluency task, showed activation of the left superior parietal cortex. Right frontal activation during testing of continuous performance also has been reported. ${ }^{40}$ These studies' determined $\mathrm{HbO}$ and $\mathrm{HbR}$ timecourse changes in the relevant activated cerebral cortices are consistent with the present results; that is, during cortical activation, $\mathrm{HbO}$ increases and HbR decreases.

Classification as a process for distinguishing data classes ${ }^{41}$ entails two steps: feature selection and classifier execution. ${ }^{42}$ As features for distinguishing different stimuli, the mean, peak, slope, kurtosis, and skewness values of $\mathrm{HbO}$ signals for individual trials, as averaged over all channels, have been adopted. As a classification algorithm, the linear discriminant analysis (LDA) technique that separates data into two or more classes have been widely utilized in various fNIRS applications involving the detection of drowsiness ${ }^{43}$ and mental workload, ${ }^{44}$ as well as in a hybrid brain-computer interface (BCI) applications using both fNIRS and EEG. ${ }^{45}$ The usability of these algorithms has been well established in the literature; see, for example, relevant review papers ${ }^{46,47}$ and multi-class problems. ${ }^{48,49}$

In the present study, fNIRS is applied to detect the visual cortex's HR to primary RGB-color stimuli, and the correlation between those responses and the various stimuli are explored. Noise-removal preprocessing and statistical analysis are used to enhance the classification accuracy. Then, the $\mathrm{HbO}$ signal is used via the three-class classifier LDA employing the mean, peak, slope, skewness, and kurtosis values as features to distinguish the RGB colors. The best two-feature combination is turned out to be peak and skewness. This indicates significantly that the colors detected by fNIRS can be differentiated using the above-mentioned features. To the best of our knowledge, this is the first fNIRS work on color detection in the human visual cortex.

\section{Materials and Methods}

\subsection{Subjects}

A total of 14 healthy volunteers (10 males and 4 females, aged 24-31 years, two left-handed) participated in the experiment. None of them had any history of neurological disorder. All of them were informed about the experiment and provided a written consent according to the latest Declaration of Helsinki. During the experiment, the subjects were asked to sit comfortably and remain motionless as much as possible. A projector screen was
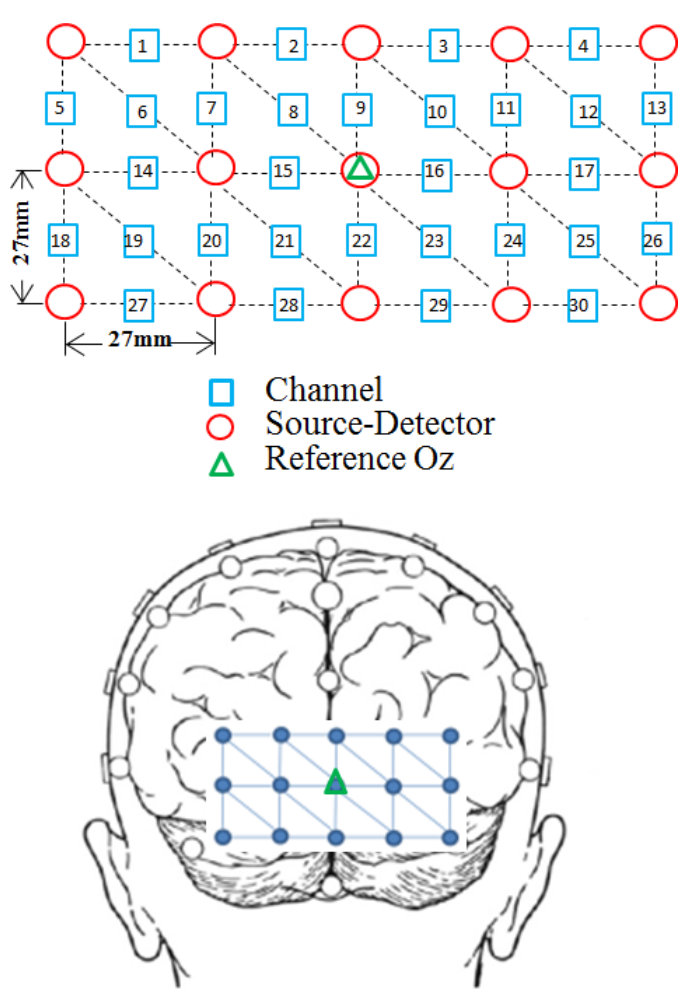

Fig. 1. Optodes configuration: The numbers represent the measurement channels; $\mathrm{Oz}$ refers to a reference point in the International 10-20 System.

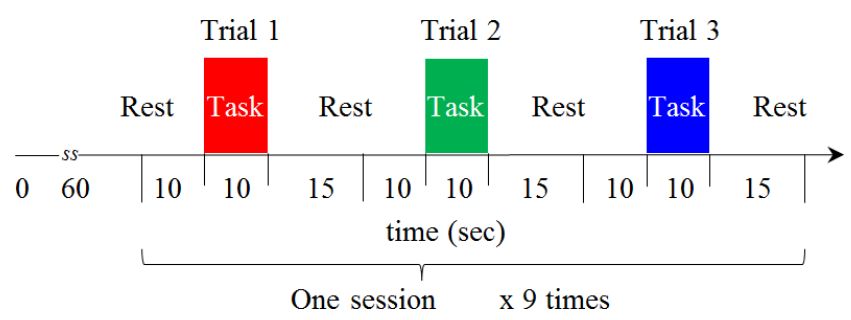

Fig. 2. Experimental RGB-color paradigm: One trial consists of a $10 \mathrm{~s}$ rest, a $10 \mathrm{~s}$ color stimulation, and a $15 \mathrm{~s}$ rest; three continuous trials of each color stimulation constitutes one session; one experiment consists of nine sessions (total time $947 \mathrm{~s}$ ). 


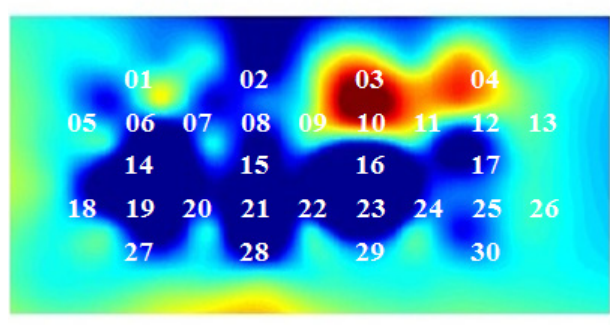

(a)

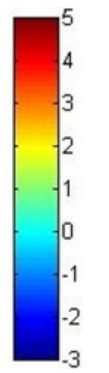

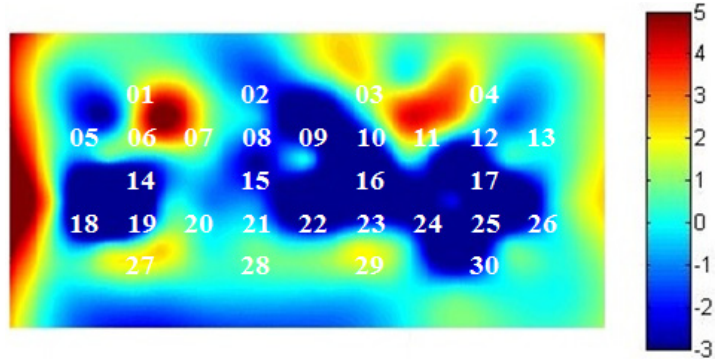

(b)

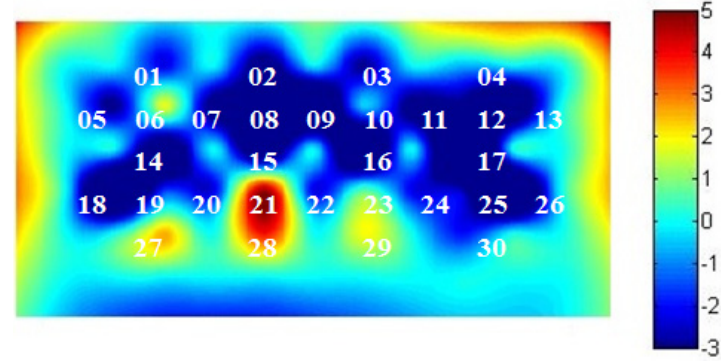

(c)

Fig. 3. Brain activation map ( $t$-map) of the primary RGB colors displayed on a white screen beamed by a projector (averaged over 14 subjects). (a) Red-color stimulation, (b) Green-color stimulation and (c) Blue-color stimulation.

placed in front of them at a distance of approximately $1 \mathrm{~m}$. The subjects were asked to keep their eyes during both the test and rest periods. Also, they were instructed to keep their blinking to the minimal level.

\section{2. fNIRS data}

The data were acquired with a continuouswave NIRS imaging system (Dynamic Near-infrared Optical Tomography (DYNOT); NIRx Medical
Technologies, USA) at a sampling rate of $1.81 \mathrm{~Hz}$. The system emits laser light at different wavelengths $(760 \mathrm{~nm}$ and $830 \mathrm{~nm}$ ) from each source. The optodes configuration illustrated in Fig. 1 indicates the channel distribution and measurement locations. The distance between adjacent optodes is $27 \mathrm{~mm}$. The source-emitter pairs are positioned above the visual cortex (the $\mathrm{Oz}$ position, according to the international 10-20 system). During the experiment, all lights were eliminated in order to minimize signal contamination. The measured

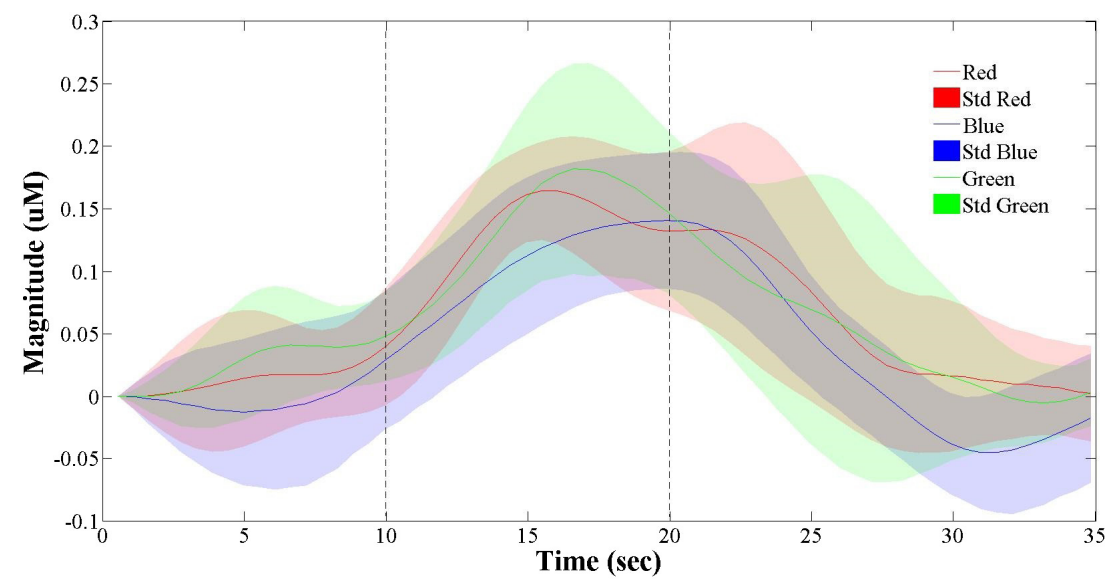

Fig. 4. The HbOs averaged over 14 subjects and their standard deviations for RGB-color stimuli. 

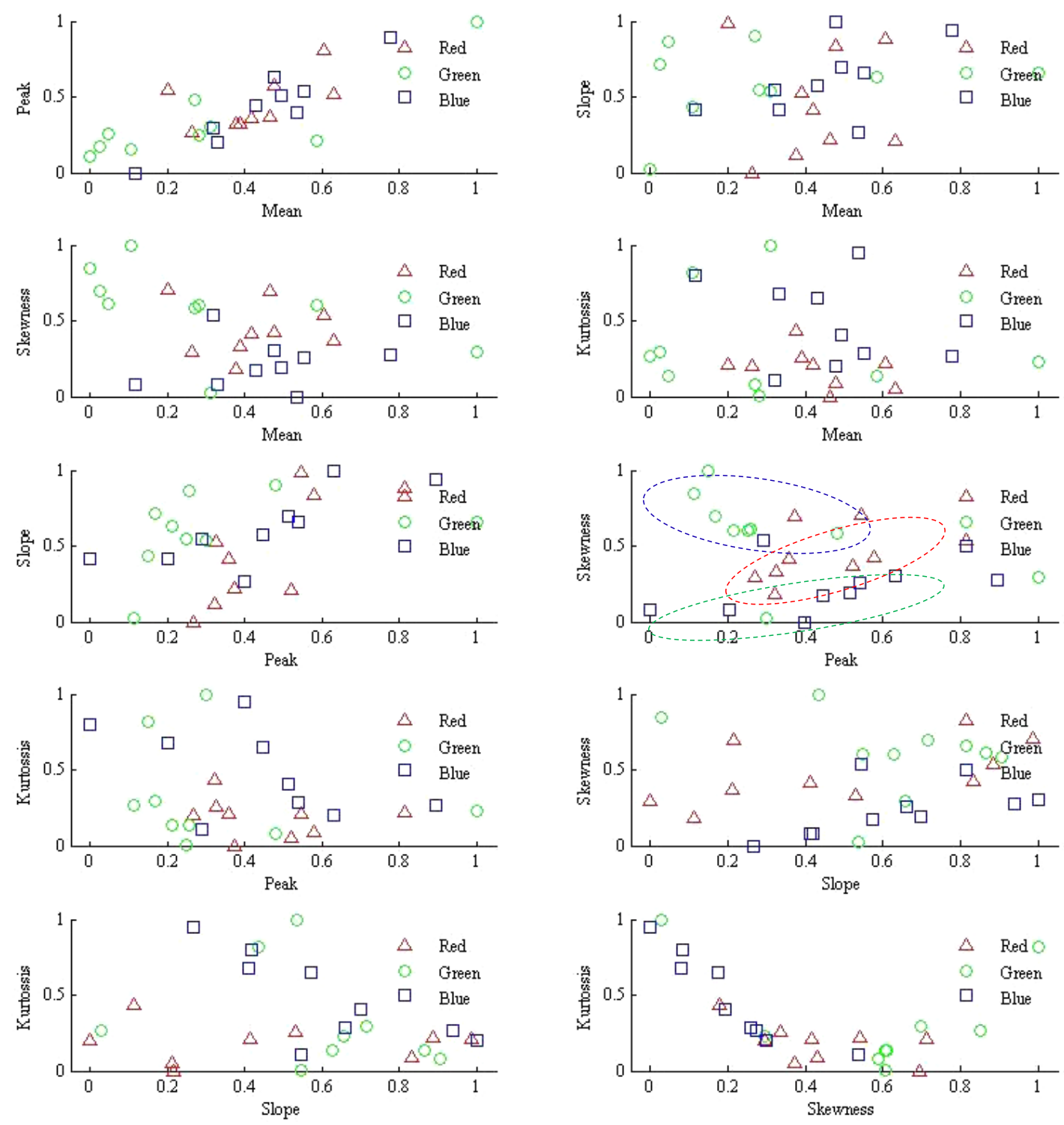

Fig. 5. Feature spaces in combination of mean, peak, slope, skewness, and kurtosis for distinguishing RGB-color stimuli (Subject 1): The triangle represents red-color the circle represents green-color and the square represents blue-color.

intensity data of the two wavelengths were converted to relative $\mathrm{HbO}$ and $\mathrm{HbR}$ concentration changes using the modified Beer-Lambert law given by $^{50-52}$

$$
\begin{aligned}
{\left[\begin{array}{l}
\Delta c_{\mathrm{HbO}}(t) \\
\Delta c_{\mathrm{HbR}}(t)
\end{array}\right]=} & {\left[\begin{array}{ll}
\alpha_{\mathrm{HbO}}\left(\lambda_{1}\right) & \alpha_{\mathrm{HbR}}\left(\lambda_{1}\right) \\
\alpha_{\mathrm{HbO}}\left(\lambda_{2}\right) & \alpha_{\mathrm{HbR}}\left(\lambda_{2}\right)
\end{array}\right]^{-1} } \\
& \times\left[\begin{array}{l}
\Delta A\left(t, \lambda_{1}\right) \\
\Delta A\left(t, \lambda_{2}\right)
\end{array}\right] \frac{1}{l \times d},
\end{aligned}
$$

where $\Delta A$ is the optical density variation of the light emitted at wavelength $\lambda_{j}, \alpha_{\mathrm{Hb} X}\left(\lambda_{j}\right)$ is the extinction coefficient of $\mathrm{Hb} X$ in $\mu \mathrm{M}^{-1} \mathrm{~mm}^{-1}, d$ is the unit-less differential pathlength factor (DPF), and $l$ is the emitter-detector distance (in $\mathrm{mm}$ ). In this study, constant values of DPF $\left(d_{1}=7.15\right.$ for $\lambda_{1}=760 \mathrm{~nm}$ and $d_{2}=5.98$ for $\lambda_{2}=830 \mathrm{~nm}$ ) were used for all channels. For signal analysis, the open-source software NIRS-SPM ${ }^{50,53,54}$ was utilized in the authors' own MATLAB ${ }^{\mathrm{TM}}$ (MathWorks, USA) code.

\subsection{Visual stimuli}

Figure 2 provides the experimental paradigm used in this work. As indicated, one task block consists of each display of RGB colors, and the rest block 


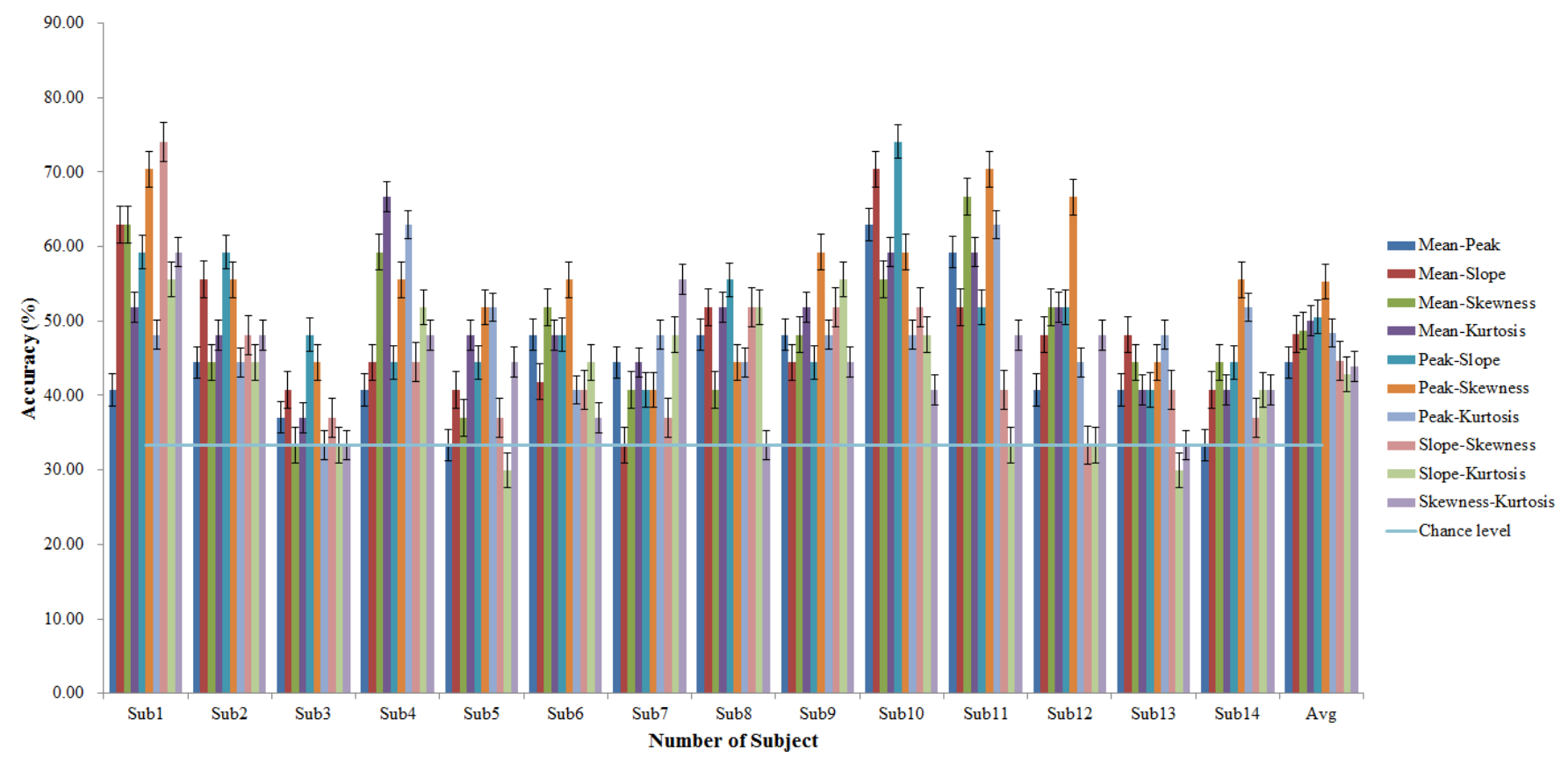

Fig. 6. Classification accuracies across 14 subjects based on 10 feature-combinations (mean, peak, slope, skewness, and kurtosis).

displays the black screen. The used RGB colors are the primary RGB colors generated by a beam projector (EPSON Model: EB-1915), whose hex and decimal codes are: Red (\#FF0000/255, 0, 0), Green (\#00FF00/0, 255, 0), and Blue $(\# 0000 \mathrm{FF} / 0,0$, 255). The duration of each color stimulus and rest period are $10 \mathrm{~s}$ and $25 \mathrm{~s}$, respectively. One session includes three repetitions of RGB stimuli. Nine sessions in total were performed; accordingly, the entire fNIRS recording duration was $16 \mathrm{~min}$.

\subsection{Signal preprocessing}

In this study, three methods (noise filtering, signal de-trending, signal normalization) were applied in the preprocessing process. First, the respiration

Table 1. Classification accuracies of 14 subjects and 10 combinations (mean, peak, slope, skewness, and kurtosis).

\begin{tabular}{lrrrrrrrrrr}
\hline No. & $\begin{array}{c}\text { Mean- } \\
\text { Peak }\end{array}$ & $\begin{array}{c}\text { Mean- } \\
\text { Slope }\end{array}$ & $\begin{array}{c}\text { Mean- } \\
\text { Skewness }\end{array}$ & $\begin{array}{c}\text { Mean- } \\
\text { Kurtosis }\end{array}$ & $\begin{array}{c}\text { Peak- } \\
\text { Slope }\end{array}$ & $\begin{array}{c}\text { Peak- } \\
\text { Skewness }\end{array}$ & $\begin{array}{c}\text { Peak- } \\
\text { Kurtosis }\end{array}$ & $\begin{array}{c}\text { Slope- } \\
\text { Skewness }\end{array}$ & $\begin{array}{c}\text { Slope- } \\
\text { Kurtosis }\end{array}$ & $\begin{array}{c}\text { Skewness- } \\
\text { Kurtosis }\end{array}$ \\
\hline Sub1 & 40.74 & 62.96 & 62.96 & 51.85 & 59.25 & 70.37 & 48.15 & 74.07 & 55.56 & 59.26 \\
Sub2 & 44.44 & 55.56 & 44.44 & 48.15 & 59.25 & 55.56 & 44.44 & 48.15 & 44.44 & 48.15 \\
Sub3 & 37.04 & 40.74 & 33.33 & 37.03 & 48.15 & 44.44 & 33.33 & 37.04 & 33.33 & 33.33 \\
Sub4 & 40.74 & 44.44 & 59.26 & 66.67 & 44.44 & 55.56 & 62.96 & 44.44 & 51.85 & 48.15 \\
Sub5 & 33.33 & 40.74 & 37.04 & 48.15 & 44.44 & 51.85 & 51.85 & 37.04 & 29.93 & 44.44 \\
Sub6 & 48.15 & 41.85 & 51.85 & 48.15 & 48.15 & 55.56 & 40.74 & 40.74 & 44.44 & 37.04 \\
Sub7 & 44.44 & 33.33 & 40.74 & 44.44 & 40.74 & 40.74 & 48.15 & 37.04 & 48.15 & 55.56 \\
Sub8 & 48.15 & 51.85 & 40.74 & 51.85 & 55.56 & 44.44 & 44.44 & 51.85 & 51.85 & 33.33 \\
Sub9 & 48.15 & 44.44 & 48.15 & 51.85 & 44.44 & 59.26 & 48.15 & 51.85 & 55.56 & 44.44 \\
Sub10 & 62.96 & 70.37 & 55.56 & 59.26 & 74.07 & 59.26 & 48.15 & 51.85 & 48.15 & 40.74 \\
Sub11 & 59.26 & 51.85 & 66.67 & 59.26 & 51.85 & 70.37 & 62.96 & 40.74 & 33.33 & 48.15 \\
Sub12 & 40.74 & 48.15 & 51.85 & 51.85 & 51.85 & 66.67 & 44.44 & 51.85 & 33.33 & 48.15 \\
Sub13 & 40.74 & 48.15 & 44.44 & 40.74 & 40.74 & 44.44 & 48.15 & 40.74 & 33.33 & 33.33 \\
Sub14 & 40.74 & 40.74 & 44.44 & 40.74 & 44.44 & 55.55 & 51.85 & 37.04 & 40.74 & 40.74 \\
Avg & 44.97 & 48.23 & 48.68 & 50.00 & 50.53 & 55.29 & 48.41 & 46.03 & 43.14 & 43.92 \\
Std & \pm 8.07 & \pm 9.79 & \pm 9.84 & \pm 8.06 & \pm 9.14 & \pm 9.58 & \pm 7.75 & \pm 10.14 & \pm 9.11 & \pm 8.07 \\
\hline
\end{tabular}


$(0.033 \mathrm{~Hz})$ and cardiac noises $(1 \mathrm{~Hz})$ contained in HRs were removed by a low-pass filter working at a cut-off frequency $0.15 \mathrm{~Hz}$. Second, the trend of the signal (e.g., low-frequency drift) was removed from the time-series data using the de-trending technique $^{55-57}$ (the detrend function in MATLAB ${ }^{\mathrm{TM}}$ ). Third, the MATLAB ${ }^{\mathrm{TM}}$ function mat2 gray was used to normalize the signal data within the $0-1$ range.

\subsection{Activation map}

Plotting of the cortical activation map is the most important step in fNIRS data analysis. This map is an intuitive display of brain cortex activity. The $t$-value-based activation map represents the ratio of the weighting coefficient to the modeled HR. A high $t$-value indicates that the measured signal is highly correlated with the modeled HR. In the present study, the $t$-values were calculated for the averaged trials of individual color stimuli using the robustfit function available in MATLAB ${ }^{\mathrm{TM}} .{ }^{56-58}$ Figure 3 shows activation maps based on RGBcolor stimuli average over 14 subjects.

\subsection{Feature selection and classification}

For feature selection and classification, we used the $\mathrm{HbO}$ signals averaged over the channels whose $t$-value was higher than a critical value, which was set to $1.6736 .^{57}$ To improve the classification accuracy and to reduce the classification time, the scheme whereby only a $2-7 \mathrm{~s}$ window within the overall 10-s task period was adopted. ${ }^{22}$ Then, the mean, peak, slope, skewness, and kurtosis values were computed. The mean was obtained by averaging of $5 \mathrm{~s}$ window data points; the slope was calculated using the polyfit function in Matlab ${ }^{\mathrm{TM}}$, and the skewness and kurtosis were calculated using Matlab ${ }^{\mathrm{TM}}$ 's skewness and kurtosis functions. In the classification, we used the LDA classifier, which is to say the classify function available in Matlab ${ }^{\mathrm{TM}}$, to verify the individual $\mathrm{HbO}$ signals. ${ }^{22,54,58-62}$

\section{Results}

Figure 3 is the activation map averaged over the 14 subjects. The obtained $t$-values are displayed in a color scale for more effective illustration of regional activation. The color bar indicates the signal intensity and the color in a pixel represents the pixel's $t$-value, respectively. Figure 4 plots the HbOs averaged over 14 subjects with the standard deviations for the RGB-color stimuli. It clearly differentiates the peak values for individual color stimuli: the maximum peak value, 0.1816 , is the green-color stimulus; 0.1642 is the red-color stimulus; the smallest peak value, 0.1405 , is the blue-color stimulus. Figure 5 shows the 2D feature spaces representing all of the two-feature combinations among the mean, peak, slope, skewness and kurtosis, respectively, for Subject 1. Figure 6 and Table 1 plot and list, respectively, the subjects' two feature combination classification accuracies as averaged over all activation channels and trials. All, notably, were above the chance level (33.33\%). The best averaged accuracy, $55.29 \%$, was achieved with the peak-skewness feature combination for 14 data (Fig. 7).

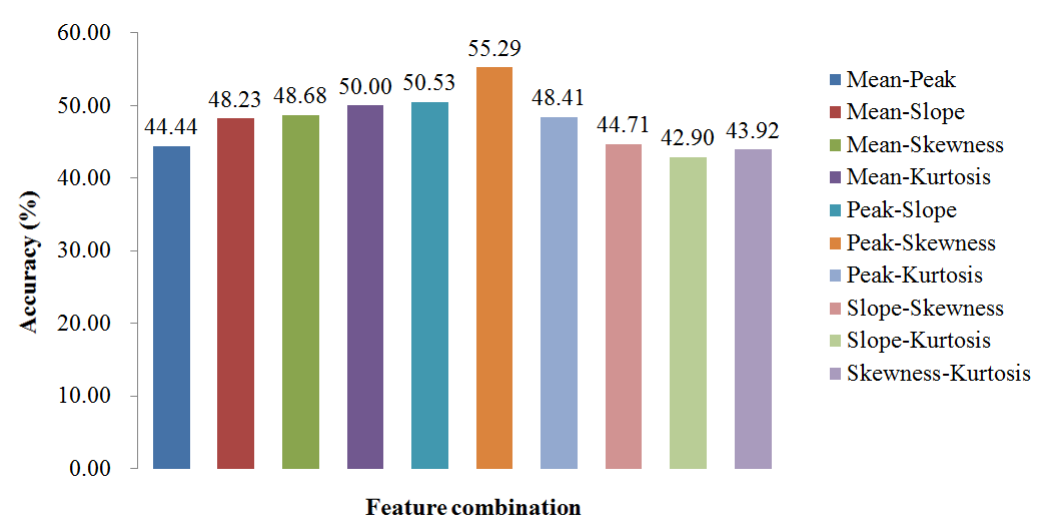

Fig. 7. Classification accuracies of various feature combinations (averaged over 14 subjects). 


\section{Discussion}

The fNIRS technique was employed, in this study, owing to its high potential as a neuro-imaging tool. Specifically, it was utilized to examine the brain activities' spatial- and temporal-distribution information. Figure 3 depicts, in the form of a $t$-map, the spatial distribution together with the expected HR induced by a given stimulus (i.e., RGB-color stimulus). ${ }^{63-65}$ However, as the signal strength in the target brain area cannot be revealed on a $t$-map, and the temporal magnitude of the HR must also be taken into account.

Also, in Fig. 3, evident are the brain activation differences according to the three RGB-color stimuli. Figure 3(a) depicts that the red-color stimulus induces more activity in the upper-right visual cortex; Fig. 3(b) indicates that upon green color stimulus there is brain activity on both sides of the visual cortex, but more on the right side than on the left; Fig. 3(c) shows that whereas there is no activity on the right side, there are, in fact, a number of active channels in the lower-left visual cortex. The peak HR values plotted in Fig. 4, meanwhile, distinguish the RGB-color stimuli as well: 0.1816 (green), 0.1642 (red), and 0.1405 (blue).

The intuitive results plotted in Fig. 7 show the peak-skewness combination to be the best feature selection for RGB-color classification (highest accuracy: $55.29 \%$ ). Although the HR and, thus, the classification accuracies varied by subjects (due to individual differences), none of the subjects showed an average classification accuracy below the chance level. Variations in classification accuracy potentially can be reduced using the optimum activation period under better environmental conditions to increase the number of trials.

There were some limitations observed during the experimental study. First, potential anomalies were evoked by sensory activity and attention/anticipation. Eye blinking, for example, incurs fluctuation in neuronal activity and peripheral HR. Second, subjects' hair generates noise in the data. In order to compensate for this problem, we recruited subjects with short hair. Taking these issues into account and avoiding them will further improve experimental results.

\section{Conclusion}

This study investigated the feasibility of detecting the visual cortex responses to RGB-color stimuli using fNIRS. RGB colors were detected by monitoring the visual cortex activity by means of $t$-map and five features (the mean, peak, slope, skewness, and kurtosis values). The $t$-map results showed the right visual cortex to be more active during the redand green-color stimuli and the left visual cortex to be more active during the blue-color stimulus. According to the three-class classification results, all of the average classification accuracies were above the chance level $(33.33 \%)$, and the highest accuracy, for Subject 1, was $74.07 \%$. The best two feature combination was the peak-skewness combination, which yielded the highest accuracy, 55.29 $\pm 9.58 \%$, as averaged over 14 subjects. The overall results suggest that fNIRS-based detection of RGB-color stimuli in the human visual cortex is feasible.

\section{Acknowledgments}

This work was supported by the China Scholarship Council (CSC) and the Convergence Technology Development Program for Bionic Arm through the National Research Foundation of Korea under the auspices of the Ministry of Science, ICT \& Future Planning, Republic of Korea (grant no. 2016M3C1B2912986).

\section{References}

1. Y. P. Xiao, Y. Wang, D. J. Felleman, "A spatially organized representation of colour in macaque Cortical Area V2," Nature 421, 535-539 (2003).

2. H. D. Lu, A. W. Roe, "Functional organization of color domains in V1 and V2 of macaque monkey revealed by optical imaging," Cereb. Cortex 18, 516-533 (2008).

3. H. Tanigawa, H. D. Lu, A. W. Roe, "Functional organization for color and orientation in macaque V4," Nat. Neurosci. 13, 1542-U135 (2010).

4. E. Goddard, D. J. Mannion, J. S. McDonald, S. G. Solomon, C. W. Clifford, "Combination of subcortical color channels in human visual cortex," J. Vision 10, 25 (2010).

5. G. J. Brouwer, D. J. Heeger, "Decoding and reconstructing color from responses in human visual cortex," J. Neurosci. 29, 13992-4003 (2009).

6. L. M. Parkes, J. B. C. Marsman, D. C. Oxley, J. Y. Goulermas, S. M. Wuerger, "Multivoxel fMRI analysis of color tuning in human primary visual cortex," J. Vision 9, 1 (2009).

7. K. T. Mullen, S. O. Dumoulin, K. L. McMahon, G. I. de Zubicaray, R. F. Hess, "Selectivity of human 
retinotopic visual cortex to s-cone-opponent, L/Mcone-opponent and achromatic stimulation," Eur. J. Neurosci. 25, 491-502 (2007).

8. K. T. Mullen, D. H. Chang, R. F. Hess, "The selectivity of responses to red-green colour and achromatic contrast in the human visual cortex: An fMRI adaptation study," Eur. J. Neurosci. 42, 2923-2933 (2015).

9. B. Laeng, K. Hugdahl, K. Specht, "The neural correlate of colour distances revealed with competing synaesthetic and real colours," Cortex 47, 320-331 (2011).

10. I. Kuriki, P. Sun, K. Ueno, K. Tanaka, K. Cheng, "Hue selectivity in human visual cortex revealed by functional magnetic resonance imaging," Cerb. Cortex 25, 4869-4884 (2015).

11. Z. Tang, H. Zhang, "To judge what color the subject watched by color effect on brain activity," Int. J. Comput. Sci. Netw. Secur. 11, 80-83 (2011).

12. S. Rasheed, D. Marini, "Classification of EEG signals produced by RGB colour stimuli," J. Biomed. Eng. Med. Imag. 2, 56 (2015).

13. E. Alharbi, S. Rasheed, S. Buhari, "Single trial classification of evoked EEG signals due to RGB colors," Brain-Broad Res. Artif. Intell. Neurosci. 7, 29-41 (2016).

14. D. A. Boas, A. M. Dale, M. A. Franceschini, "Diffuse optical imaging of brain activation: Approaches to optimizing image sensitivity, resolution, and accuracy," Neuroimage 23, S275-S288 (2004).

15. M. Wolf, M. Ferrari, V. Quaresima, "Progress of near-infrared spectroscopy and topography for brain and muscle clinical applications," J. Biomed. Opt. 12, 062-104 (2007).

16. X.-S. Hu, K.-S. Hong, S. S. Ge, "Recognition of stimulus-evoked neuronal optical response by identifying chaos levels of near-infrared spectroscopy time series," Neurosci. Lett. 504, 115-120 (2011).

17. A. C. Merzagora, M. T. Schultheis, B. Onaral, M. Izzetoglu, "Functional near-infrared spectroscopybased assessment of attention impairments after traumatic brain injury," J. Innov. Opt. Health Sci. 4, 251-260 (2011).

18. M. J. Khan, X. Liu, M. R. Bhutta, K.-S. Hong, Drowsiness detection using fNIRS in different time windows for a passive BCI, In Biomedical Robotics and Biomechatronics (BioRob), 2016 6th IEEE Int. Conf. IEEE (2016), pp. 227-231.

19. M. Ferrari, V. Quaresima, "A brief review on the history of human functional near-infrared spectroscopy (fNIRS) development and fields of application," Neuroimage 63, 921-935 (2012).

20. S. Koehler, J. Egetemeir, P. Stenneken, S. P. Koch, P. Pauli, A. J. Fallgatter, M. J. Herrmann, "The human execution/observation matching system investigated with a complex everyday task: A functional near-infrared spectroscopy (fNIRS) Study," Neurosci. Lett. 508, 73-77 (2012).

21. H. Meiri, I. Sela, P. Nesher, M. Izzetoglu, K. Izzetoglu, B. Onaral, Z. Breznitz, "Frontal lobe role in simple arithmetic calculations: An fNIRS study," Neurosci. Lett. 510, 43-7 (2012).

22. N. Naseer, K.-S. Hong, "Classification of functional near-infrared spectroscopy signals corresponding to the right- and left-wrist motor imagery for development of a brain-computer interface," Neurosci. Lett. 553, 84-89 (2013).

23. L. Zhang, J. Y. Sun, B. L. Sun, C. Y. Gao, H. Gong, "Detecting bilateral functional connectivity in the prefrontal cortex during a stroop task by near-infrared spectroscopy," J. Innov. Opt. Health. Sci. 6, 1350031 (2013).

24. M. R. Bhutta, K.-S. Hong, B. M. Kim, M. J. Hong, Y. H. Kim, S. H. Lee, "Note: Three wavelengths near-infrared spectroscopy system for compensating the light absorbance by water," Rev. Sci. Instrum. 85, 026111 (2014).

25. J. M. Kainerstorfer, A. Sassaroli, M. L. Pierro, B. Hallacoglu, S. Fantini, "Coherent hemodynamics spectroscopy based on a paced breathing paradigmrevisited," J. Innov. Opt. Health. Sci. 7, 145013 (2014).

26. N. D. Thang, V. V. Toi, L. G. Tran, N. H. M. Tam, L. A. Trinh, "Investigation of human visual cortex responses to flickering light using functional near infrared spectroscopy and constrained ICA," J. Innov. Opt. Health Sci. 7, 1450031 (2014).

27. M. R. Bhutta, M. J. Hong, Y. H. Kim, K.-S. Hong, "Single-trial lie detection using a combined fNIRSpolygraph system," Front. Psychol. 6, 709 (2015).

28. K.-S. Hong, N. Naseer, Y. H. Kim, "Classification of prefrontal and motor cortex signals for three-class fNIRS-BCI," Neurosci. Lett. 587, 87-92 (2015).

29. T. Li, Y. Li, Y. L. Sun, M. X. Duan, L. Y. Peng, "Effect of head model on monte carlo modeling of spatial sensitivity distribution for functional nearinfrared spectroscopy," J. Innov. Opt. Health Sci. 8, 1550024 (2015).

30. N. Naseer, K.-S. Hong, "Decoding answers to fourchoice questions using functional near infrared spectroscopy," J. Near Infrared Spectrosc. 23, 2331 (2015).

31. D. P. Pinero, B. Monllor, V. Moncho, V. J. Camps, D. de Fez, "Visual function alterations in essential tremor: A case report," J. Innov. Opt. Health. Sci. 8 , 1550040 (2015).

32. A. Sassaroli, J. Kainerstorfer, S. Fantini, "Study of capillary transit time distribution in coherent hemodynamics spectroscopy," J. Innov. Opt. Health Sci. 8, 1550025 (2015). 
33. M. Shokoufi, F. Golnaraghi, "Development of a handheld diffuse optical breast cancer assessment probe," J. Innov. Opt. Health Sci. 9, 1650007 (2016).

34. V. Y. Toronov, X. F. Zhang, A. G. Webb, "A spatial and temporal comparison of hemodynamic signals measured using optical and functional magnetic resonance imaging during activation in the human primary visual cortex," Neuroimage 34, 1136-1148 (2007).

35. X. S. Hu, K.-S. Hong, S. S. Ge, M. Y. Jeong, "Kalman estimator- and general linear model-based on-line brain activation mapping by near-infrared spectroscopy," Biomed. Eng. Online 9, 82 (2010).

36. X. S. Hu, K.-S. Hong, S. S. Ge, "fNIRS-based online deception decoding," J. Neural Eng. 9, 026012 (2012).

37. R. L. Barbour, H. L. Graber, Y. Pei, S. Zhong, C. H. Schmitz, "Optical tomographic imaging of dynamic features of dense-scattering media," J. Opt. Soc. Am. A-Opt. Image. Sci. Vis. 18, 3018-3036 (2001).

38. M. Ferrari, L. Mottola, V. Quaresima, "Principles, techniques, and limitations of near infrared spectroscopy," Can. J. Appl. Physiol. 29, 463-487 (2004).

39. A. Villringer, J. Planck, C. Hock, L. Schleinkofer, U. Dirnagl, "Near infrared spectroscopy (NIRS): A new tool to study hemodynamic changes during activation of brain function in human adults," Neurosci. Lett. 154, 101-104 (1993).

40. A. J. Fallgatter, M. Roesler, L. Sitzmann, A. Heidrich, T. J. Mueller, W. K. Strik, "Loss of functional hemispheric asymmetry in alzheimer"s dementia assessed with near-infrared spectroscopy," Cognit. Brain Res. 6, 67-72 (1997).

41. R. O. Duda, P. E. Hart, D. G. Stork, Pattern Classification, John Wiley \& Sons (2012).

42. H. Liu, L. Yu, "Toward integrating feature selection algorithms for classification and clustering," IEEE Trans. Knowl. Data. Eng. 17, 491-502 (2005).

43. M. J. Khan, K.-S. Hong, "Passive BCI based on drowsiness detection: An fNIRS study," Biomed. Opt. Express 6, 4063-4078 (2015).

44. C. Herff, D. Heger, O. Fortmann, J. Hennrich, F. Putze, T. Schultz, "Mental workload during N-back task-quantified in the prefrontal cortex using fNIRS," Front. Hum. Neurosci. 7, 935 (2014).

45. F. Putze, S. Hesslinger, C. Y. Tse, Y. Y. Huang, C. Herff, C. T. Guan, T. Schultz, "Hybrid fNIRS-EEG based classification of auditory and visual perception processes," Front. Neurosci. 8, 373 (2014).

46. F. Lotte, M. Congedo, A. Lecuyer, F. Lamarche, B. Arnaldi, "A review of classification algorithms for EEG-based brain-computer interfaces," J. Neural Eng. 4, R1-R13 (2007).
47. F. Pereira, T. Mitchell, M. Botvinick, "Machine learning classifiers and fMRI: A tutorial overview," Neuroimage 45, S199-S209 (2009).

48. D. Garrett, D. A. Peterson, C. W. Anderson, M. H. Thaut, "Comparison of linear, nonlinear, and feature selection methods for EEG signal classification," IEEE Trans. Neural Syst. Rehabil. Eng. 11, 141-144 (2003).

49. A. Schlogl, F. Lee, H. Bischof, G. Pfurtscheller, "Characterization of four-class motor imagery EEG data for the BCI-competition," J. Neural Eng. 2, L14-L22 (2005).

50. J. Chul, S. Tak, K. E. Jang, J. Jung, J. Jang, "NIRS-SPM: Statistical parametric mapping for near-infrared spectroscopy," Neuroimage 44, 428447 (2009).

51. M. A. Kamran, K.-S. Hong, "Linear parametervarying model and adaptive filtering technique for detecting neuronal activities: An fNIRS study," J. Neural. Eng. 10, 056002 (2013).

52. H. Santosa, M. J. Hong, S. P. Kim, K.-S. Hong, "Noise reduction in functional near-infrared spectroscopy signals by independent component analysis," Rev. Sci. Instrum. 84, 073106 (2013).

53. K.-S. Hong, H. D. Nguyen, "State-space models of impulse hemodynamic responses over motor, somatosensory, and visual cortices," Biomed. Opt. Express 5, 1778-1798 (2014).

54. M. J. Khan, M. J. Hong, K.-S. Hong, "Decoding of four movement directions using hybrid NIRS-EEG brain-computer interface," Front. Hum. Neurosci. 8, 244 (2014).

55. J. Tanabe, D. Miller, J. Tregellas, R. Freedman, F. G. Meyer, "Comparison of detrending methods for optimal fMRI preprocessing," Neuroimage 15, 902907 (2002).

56. H. Santosa, M. J. Hong, K.-S. Hong, "Lateralization of music processing with noises in the auditory cortex: An fNIRS study," Front. Behav. Neurosci. 8, 418 (2014).

57. K.-S. Hong, H. Santosa, "Decoding four different sound-categories in the auditory cortex using functional near-infrared spectroscopy," Hear. Res. 333, 157-166 (2016).

58. X. Liu, K.-S. Hong, fNIRS based color detection from human visual cortex, in Society of Instrument and Control Engineers of Japan (SICE), 2015 54th Annual Conf. IEEE (2015), pp. 1156-1161.

59. N. Naseer, M. J. Hong, K.-S. Hong, "Online binary decision decoding using functional near-infrared spectroscopy for the development of brain-computer interface," Exp. Brain Res. 232, 555-564 (2014).

60. N. Naseer, K.-S. Hong, "fNIRS-based brain-computer interfaces: A review," Front. Hum. Neurosci. 9, 3 (2015). 
61. K.-S. Hong, N. Naseer, "Reduction of delay in detecting initial dips from functional near-infrared spectroscopy signals using vector-based phase analysis," Int. J. Neural. Syst. 26, 1650012 (2016).

62. N. Naseer, F. M. Noori, N. K. Qureshi, K.-S. Hong, "Determining optimal feature-combination for LDA classification of functional near-infrared spectroscopy signals in brain-computer interface application," Front. Hum. Neurosci. 10, 237 (2016).

63. X.-S. Hu, K.-S. Hong, S. S. Ge, "Reduction of trialto-trial variability in functional near-infrared spectroscopy signals by accounting for resting-state functional connectivity," J. Biomed. Opt. 18, 017003 (2013).

64. H.-D. Nguyen, K.-S. Hong, "Bundled-optode implementation for 3D imaging in functional near-infrared spectroscopy," Biomed. Opt. Exp. 7, 3491-3507 (2016).

65. H.-D. Nguyen, K.-S. Hong, Y.-I Shin, "Bundledoptode method in functional near-infrared spectroscopy," PLoS ONE 10, e0165146 (2016). 